\title{
Waiting Time following Neoadjuvant Chemoradiotherapy for Rectal Cancer: Does It Really Matter?
}

\author{
Aris Plastiras $^{a} \quad$ Michail Sideris $^{a} \quad$ Andy Gaya $^{b} \quad$ Amyn Haji ${ }^{a}$ \\ Joseph Nunoo-Mensah ${ }^{\mathrm{a}}$ Asif Haq $^{\mathrm{a}}$ Savvas Papagrigoriadis ${ }^{\mathrm{a}}$ \\ a King's College Hospital and 'buy's and St. Thomas' Hospital, NHS Foundation Trust, \\ London, UK
}

\section{Keywords}

Colorectal cancer - Improvement of prognosis - Neoadjuvant chemoradiotherapy ·

Therapeutic management

\begin{abstract}
Background: Neoadjuvant chemoradiotherapy (CRT) is considered the standard approach before any surgical intervention for locally advanced rectal tumors and has been proven to significantly improve the local recurrence rates of rectal cancer. However, the optimal timing of surgical resection after neoadjuvant CRT remains debatable. Objective and Methods: We conducted a retrospective review of 65 consecutive patients with locally advanced rectal cancer who underwent preoperative CRT followed by surgical resection in order to evaluate the optimal time for surgical treatment. We used two alternative groups for analysis: patients who underwent surgery up to 6 weeks after CRT $(n=28)$ and those who underwent surgery 6 weeks or more after CRT $(n=27)$. Also, we compared patients who were operated on within 3 months $(n=39)$ with those who underwent surgical resection after more than 3 months $(n=16)$. Nonresponders to CRT were excluded from the analysis. Results: There was no statistically significant association between waiting period post CRT and radiological downstaging for any group ( $p>0.05$ for any association). Also, there was no association between recurrence of disease, cancer-related deaths, perineural invasion, or positive lymph node ratio and any waiting period up to 3 months ( $p>0.05$ for all associations). Conclusion: In this small exploratory study there was no evident difference in outcome according to timing of surgery, which suggests that further research in larger cohorts is warranted.




\section{Introduction}

Neoadjuvant chemoradiotherapy (CRT) is considered the standard approach before any surgical intervention for locally advanced rectal tumors [1]. Total mesorectal excision combined with neoadjuvant CRT has resulted in significant improvement of local disease control, lower rates of local recurrence, more effective tumor downstaging, and greater success rates in sphincter-sparing surgery [2-4]. Ideally, surgery should take place at the time of optimal response to neoadjuvant CRT as the effects of the latter are time dependent.

The optimal timing for rectal cancer surgery after neoadjuvant therapy still remains equivocal. Most surgeons tend to operate on the basis of a 6-week window following completion of CRT for rectal cancer. This approach was implemented into practice following the Lyon R90-01 trial in 1999, when Francois et al. [5] randomized 201 patients with the aim to proceed to radical surgery either 2 or 6 weeks after completion of RCT. The group with the longer waiting period had better tumor response, pathological downstaging, and higher sphincter-sparing surgery rates. However, there has been concern that such delays would make the operation more challenging due to technical difficulties, including fibrosis, and may result in increased morbidity or even locoregional recurrence $[1,6]$. Many surgeons remain reluctant to delay surgical intervention beyond 2 months.

Given the controversy of previous studies along with the fact that there has not been a consensus yet, we decided to conduct a retrospective cohort study in order to evaluate which would be the optimal time to operate. We compared downstaging defined by radiological and pathological TNM stage, response to CRT, recurrence rate, disease-free survival rate, and cancer-related death against the optimal time to surgery in patients with either complete and partial response or no response to multimodality treatment of rectal cancer.

\section{Materials and Methods}

We performed a retrospective review of the prospectively collected data on 65 consecutive patients with locally advanced rectal cancer who underwent preoperative CRT followed by surgical tumor resection between April 2008 and May 2014 at King's College Hospital. The inclusion criterion was radiologically staged cT1-4Nx rectal cancer using high-resolution pelvic magnetic resonance imaging and/or multislice computed tomography of the abdomen, pelvis, and chest. Carcinoembryonic antigen levels were measured as well. A multidisciplinary team panel consisting of colorectal surgeons, medical oncologists, a chemotherapy specialist, clinical oncologists, radiologists, pathologists, nursing specialists, gastroenterologists, and palliative care specialists agreed on the management of all rectal cancer patients. The indication on which deferring the surgery for more than 12 weeks was decided was mainly based on fitness criteria, i.e., the need for cardiovascular, renal, or respiratory optimization preoperatively ( $90 \%$ of cases), and occasionally ( $<10 \%$ of cases) on the surgeon's expert view. All patients received neoadjuvant CRT prior to surgical resection according to protocol and individual multidisciplinary team recommendation. More specifically, they received preoperative radiotherapy of 45-54 Gy/25 fractions to the pelvic floor. The chemotherapy regimen was capecitabine. After CRT imaging was obtained on completion of CRT as per local multidisciplinary team protocol. Surgical resection was performed by specialist colorectal surgeons in a tertiary center according to local protocols. Pelvic autonomic nerve preservation and en bloc removal of the rectal tumor and surrounding mesorectum was the standard operative practice.

For the purposes of our study, we required a system of evaluation of the radiological downstaging post CRT. As we could not identify one in the current literature, we postulated a novel evaluation system that we called "TNM ladder." More specifically, we calculated the pre-neoadjuvant stage (TNM summary based on radiological staging) and the post-pathological stage (TNM summary) difference. For instance, based on pathological downstaging, if the TNM sum in a patient before neoadjuvant CRT was 4 due to a T3N1M0 tumor $(3+1=4)$ and the TNM sum in the pathology report was 2 due to a T2N0 tumor $(2+0=2)$, it was counted as I minus 2 I in our dTNM system. Absence of response was calculated as (0) and disease progression carried a positive sign. We found this to be a novel and accurate system of quantitative evaluation of response to CRT for the purposes of our study. 
Fig. 1. Comparison of survival between the two groups $(<6$ weeks vs. $>6$ weeks).
Plastiras et al.: Waiting Time following Neoadjuvant Chemoradiotherapy for Rectal Cancer: Does It Really Matter?

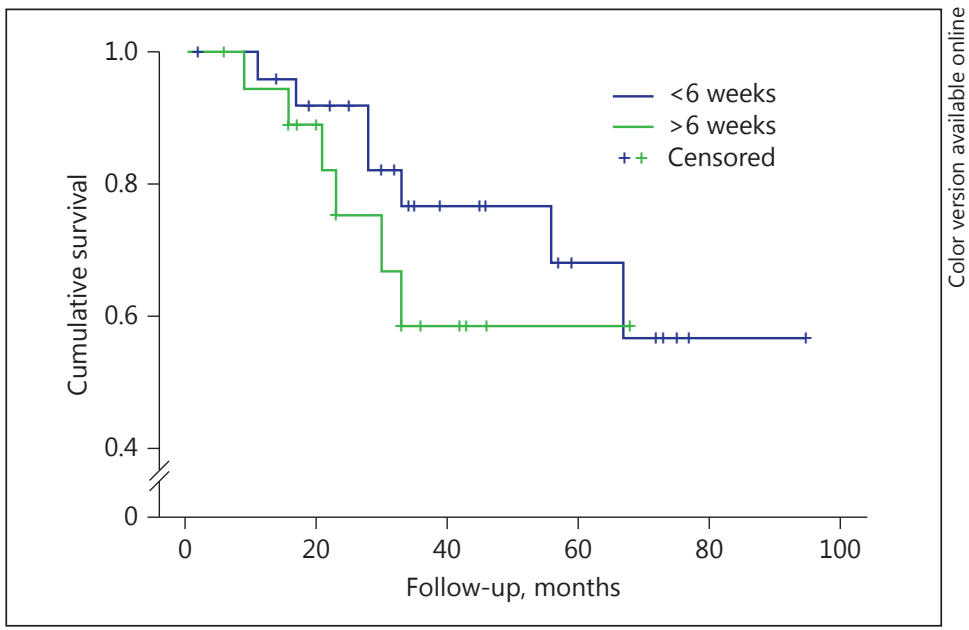

We defined outcomes as (1) the difference in the radiological TNM stage before and after (radiological downstaging) as well as (2) the difference between the radiological stage prior to treatment and the pathological TNM stage postoperatively (pathological downstaging).

Initially we split the patients into two groups. Group A included patients who were operated on up to 6 weeks $(n=28)$ and group B those who had surgery after 6 weeks or later $(n=27)$. Finally, we tried to do an alternative split of the responders into group A, which included patients who were operated on within 3 months $(n=39)$, and group B with those who underwent surgical resection later than 3 months following CRT $(n=16)$. In either group an independent $t$ test analysis was performed to assess for any significant difference in the outcomes of cancer-related death (Fig. 1), downstaging as defined by difference in the radiological and pathological u/p TNM, recurrence, perineural invasion, and positive lymph node ratio.

Data were analyzed on IBM SPSS for Macintosh version 22 (IBM Corp., Armonk, NY, USA). Univariate nonparametric descriptive statistics including Spearman rho were used to identify any potential associations. Kaplan-Meier curves were used to calculate survival.

\section{Results}

The cohort population consisted of 42 males (64.6\%) and 23 females (35.4\%) who received treatment for rectal cancer in a tertiary center. The mean age was 70.87 years (range $=32.2-90.5, \mathrm{SD}=14.84$ ). Before neoadjuvant CRT, uT1 was $1.5 \%$, uT2 7.7\%, uT3 $64.6 \%$, and uT4 27.7\%. uN0 was $n=10(15.4 \%), \mathrm{uN} 1 n=23(35.4 \%), \mathrm{uN} 2 n=31(47.7 \%)$, and uN3 $n=1(1.5 \%)$, as described in Table 1 .

Following neoadjuvant CRT, there were $n=3$ uT0 (4.6\%), $n=2$ uT1 (3.1\%), $n=20$ uT2 (30.8\%), $n=31$ uT3 (47.7\%), $n=9$ uT4 (13.8\%), $n=32$ uN0 (49.2\%), $n=18$ uN1 (32.3\%), $n=25 \mathrm{uN} 2$ (18.5\%), $n=45 \mathrm{uM0}$ (69.2\%), and $n=20 \mathrm{uM} 1$ (30.8\%). The mean difference in the step reduction (defined as preradiological TNM minus postradiological TNM) was -1.35 (range $=0-5, \mathrm{SD}=0.97$ ), and preradiological TNM minus postpathological TNM was -1.32 (range $=0-5, \mathrm{SD}=0.985)$. Ten patients $(15.4 \%)$ were classified as nonresponders, and $n=31$ (47.7\%) downstaged to $1, n=17(26.2 \%)$ downstaged to $2, n=6(9.2 \%)$ downstaged to 3 , and $n=1(1.5 \%)$ downstaged to 5 . According to the radiological TNM ladder, among the responders, $3(4.6 \%)$ had complete response and $52(80 \%)$ had partial response.

Based on pathological downstaging, $n=12(18.5 \%)$ were classified as nonresponders; $n=29(44.6 \%)$ were downstaged to $1, n=17(26.2 \%)$ were downstaged to $2, n=6(9.2 \%)$ were downstaged to 3 , and $n=1(1.5 \%)$ was downstaged to 5 in our TNM ladder. In $n=48$ patients there was no perineural invasion $(72.9 \%)$, whereas $n=8(12.0 \%)$ were perineural 
Tumors

Table 1. Demographics and cohort description

\section{Age, years \\ Sex}

Male

Female

Follow-up, months

Waiting time, months

Radiological response

No $(n=10)$

Partial $(n=52)$

Complete $(n=3)$

Cancer-related death (responders)

Yes $(n=13)$

No $(n=42)$

Recurrence analysis

Local $(n=3)$

Distal $(n=9)$

Differentiation

Well

Moderate

Poor

uT stage before CRT

T1

$\mathrm{T} 2$

T3

$\mathrm{T} 4$

uN stage before CRT

N0

N1

N2

N3

uT stage after CRT

T0

T1

T2

T3

$\mathrm{T} 4$

uN stage after CRT

NO

N1

N2

Lymph nodes

Positive lymph nodes

Positive lymph node ratio

dTNM ladder (responders)

$1(n=31)$

$2(n=17)$

$3(n=6)$

$5(n=1)$

Circumferential resection margin

Clear

Inconclusive

Lymphovascular invasion

Positive

Negative

Perineural invasion

Positive

Negative

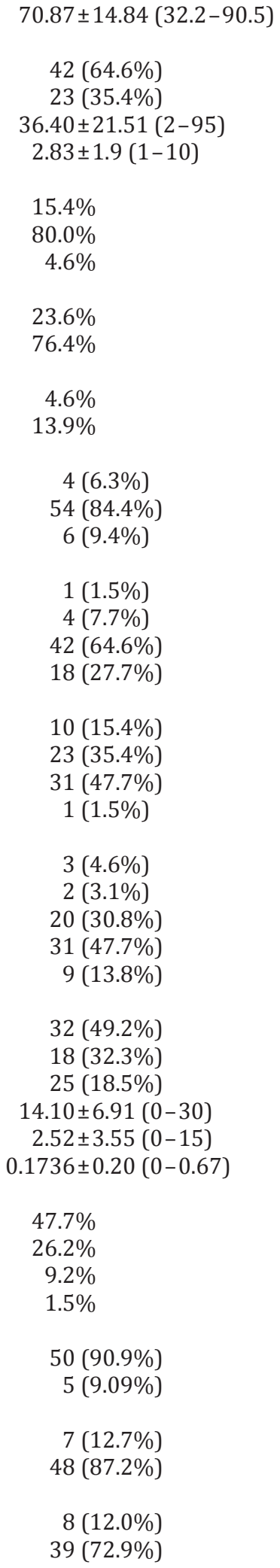

Values are presented as mean \pm SD (range), $n(\%)$, or \%. CRT, chemoradiotherapy. 
Plastiras et al.: Waiting Time following Neoadjuvant Chemoradiotherapy for Rectal Cancer: Does It Really Matter?

Table 2. Comparison of outcomes (cancer-related death, recurrence) and pathology features (perineural invasion/lymph nodes) for both groupings

\begin{tabular}{|c|c|c|c|c|c|c|}
\hline & \multicolumn{2}{|c|}{$<6$ weeks vs. $>6$ weeks } & \multicolumn{2}{|c|}{$<12$ weeks vs. $>12$ weeks } & \multicolumn{2}{|c|}{ Waiting time ${ }^{1}$} \\
\hline & $p$ value & coefficient & $p$ value & coefficient & $p$ value & coefficient \\
\hline Cancer-related death & $\begin{array}{l}0.814 \\
n=7(26 \%)\end{array}$ & $\begin{array}{l}-0.033 \\
n=6(28 \%)\end{array}$ & $\begin{array}{l}0.959 \\
n=9(24.3 \%)\end{array}$ & $\begin{array}{l}0.007 \\
n=4(25 \%)\end{array}$ & 0.851 & -0.026 \\
\hline Recurrence of disease & $\begin{array}{l}0.653 \\
n=11(40.4 \%)\end{array}$ & $\begin{array}{l}-0.063 \\
n=9(34.6 \%)\end{array}$ & $\begin{array}{l}0.216 \\
n=16(43.2 \%)\end{array}$ & $\begin{array}{l}-0.173 \\
n=4(25 \%)\end{array}$ & 0.289 & -0.148 \\
\hline \multirow[t]{2}{*}{ Downstaging } & 0.790 & 0.037 & 0.197 & 0.180 & 0.345 & 0.132 \\
\hline & $<6$ weeks & $>6$ weeks & $<12$ weeks & $>12$ weeks & & \\
\hline Mean \pm SD & $1.55 \pm 0.75$ & $1.69 \pm 0.92$ & $1.51 \pm 0.69$ & $1.87 \pm 1.08$ & & \\
\hline Perineural invasion & 0.366 & 0.136 & 0.148 & 0.217 & 0.158 & 0.212 \\
\hline Positive lymph nodes & $n=3(11.1 \%)$ & $n=4(21 \%)$ & $n=4(11.1 \%)$ & $n=3(30 \%)$ & & \\
\hline Positive LN ratio & 0.195 & 0.194 & 0.678 & -0.063 & 0.623 & 0.074 \\
\hline Mean \pm SD & $2.11 \pm 3.74$ & $3.05 \pm 3.76$ & $2.52 \pm 3.56$ & $2.40 \pm 4.52$ & & \\
\hline
\end{tabular}

LN, lymph node. ${ }^{1}$ Continuous.

positive. In some cases, perineural invasion was not documented in the biopsy outcome, hence we did not know. The mean number of lymph nodes was 14.10 (range $=0-30, \mathrm{SD}=$ 6.91), and the mean number of positive lymph nodes was 2.52 (range $=0-15, \mathrm{SD}=3.55$ ). The mean positive lymph node ratio was 0.1736 (range $=0-0.67$, $\mathrm{SD}=0.20$ ). The mean waiting period prior to surgery was 2.83 months (range $=1-10, \mathrm{SD}=1.900$ ).

Local recurrence was noted in $n=3(4.6 \%)$ of our study group and distant metastasis in $n=9(13.9 \%)$. Cancer-related death was noted in $n=15$ (23.1\%). A Pearson correlation analysis between the waiting period until operation and outcomes was performed.

Initially we used an independent $t$ test to compare the mean number of positive lymph nodes for responders versus nonresponders ( 2.1 vs. $2.5, p=0.747$ ) as well as the number of total lymph nodes for responders versus nonresponders (14.5 vs. 13.3, $p=0.728$ ). Carcinoembryonic antigen was measured pre- as well as postoperatively, but results did not reach statistical significance. Therefore, we excluded nonresponders to the later stage of the analysis (group comparisons).

We split the responders into group A (patients who were operated on within 3 months) and group B (those who underwent surgical resection later than 3 months following CRT), and there was no significant association $(p>0.05$ for all associations including cancer-related death, downstaging as defined by difference in the radiological and pathological $u / p$ TNM, recurrence, perineural invasion, and positive lymph node ratio).

When we used the alternative grouping (group A: patients who were operated on up to 6 weeks; group B: those who had surgery after 6 weeks or later), there was no statistically significant difference in any of the abovementioned outcomes (Table 2; Fig. 1, 2).

\section{Discussion}

Neoadjuvant CRT has been widely used in the treatment of patients with locally advanced rectal cancer, as it has shown remarkable benefits in reducing local recurrence rates $[7,8]$ as well as improving disease-free survival time and overall survival in various randomized controlled trials [7, 9]. The Dutch Colorectal Cancer Group [4] recently showed that preoperative radiotherapy reduced 10 -year local recurrence by more than $50 \%$ relative to surgery alone, without an increase in overall survival. Park et al. [10] retrospectively reviewed 725 
Fig. 2. Comparison of survival between the two groups $(<12$ weeks vs. $>12$ weeks).
Plastiras et al.: Waiting Time following Neoadjuvant Chemoradiotherapy for Rectal Cancer: Does It Really Matter?

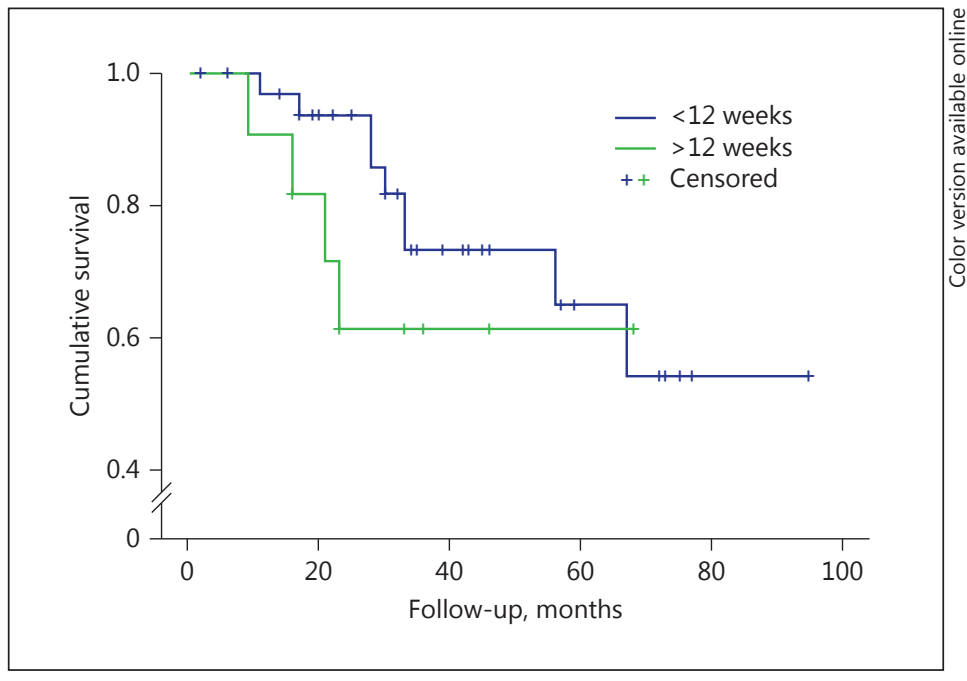

patients with locally advanced rectal cancer, treated with CRT and radical surgery, in order to assess how oncological outcomes are linked to the degree of pathological response. They concluded that response to CRT is associated with a better 5 -year disease-free survival rate as well as longer overall survival.

Even though CRT has improved the outcome of rectal cancer, the optimal timing of surgical resection after CRT remains uncertain. De Campos-Lobato et al. [1] showed that waiting for more than 8 weeks between completion of CRT and surgery was associated with significant improvement in pathological complete response (pCR) rate (30.8 vs. $16.5 \%, p=0.03)$ and decreased the 3 -year local recurrence rate (1.2 vs. $10.5 \%, p=0.04)$. Other studies have indicated downstaging and better surgical outcomes with a delay of more than 8 weeks $[11,12]$. This published evidence suggests that there is continuous local downstaging beyond 8 weeks in many patients. In addition, there are studies which showed that a waiting interval longer than 10 weeks between completion of CRT and surgical intervention is more effective. Tran et al. [13] found that patients had better tumor size reduction when operation was deferred for a few more weeks and suggested a delayed interval between CRT and surgery of 10-14 weeks. Garcia-Aguilar et al. [14] showed that prolonging the time interval between completion of CRT and total mesorectal excision (to an average of 11 weeks) may increase the pCR rate without significantly increasing CRT- or chemotherapy-related adverse events, operative difficulty, or postoperative complications, compared to traditional neoadjuvant CRT. The average delay in that study was set at 6 weeks. Tulchinsky et al. [15] found that a neoadjuvant CRT-surgery interval $>7$ weeks was associated with higher rates of pCR and near pCR (17 vs. 35\%, $p=0.03$ ), decreased recurrence, and improved disease-free survival $(p=0.05)$.

Many studies support that downstaged pathological nodal stage is associated with decreased recurrence and disease-free survival rates, and it is considered a consistent and strong predictor of survival rates [16-20]. Kim et al. [20] ran a study on the impact of pathological downstaging following irradiation versus local recurrence and survival. They observed that histopathological nodal downstaging was the most important prognostic factor for the patients. In our study we divided our patients into groups A and B based on the waiting time until surgical intervention (initially 6 weeks vs. later, and 12 weeks or later). No statistically significant correlations were defined in that study as in our retrospective study between the waiting period of time until the surgery and outcomes in terms of radiological TNM step reduction and pathological stage, perineural invasion, positive lymph node ratio, recurrence, and cancer-related death. 
In addition, Fang et al. [21] conducted a study to evaluate the benefits of delayed surgery after CRT in advanced rectal cancer regarding aspects of tumor response, survival, and its deleterious effects. They concluded that delayed surgery after CRT offered no clear benefits to those patients regarding complete response, downstaging, early postoperative complications, and local recurrence. Panagiotopoulou et al. [22] evaluated in a retrospective study whether delaying surgery following long-course CRT for rectal cancer correlated with pCR. Although no statistically significant predictors of either complete response or tumor nodal status downstaging were identified, a trend towards $\mathrm{pCR}$ was seen within the group that waited longer to have surgery following completion of long-course CRT. In another cohort study, Li et al. [23] compared the oncological outcomes of rectal cancer patients with clinical complete response who were managed according to a wait-and-see policy or with surgery. They postulated that under strict criteria a wait-and-see policy might be considered instead of surgery for rectal cancer patients with no residual tumor or involved lymph nodes after neoadjuvant CRT.

Our study is in line with previous reports on the safety of a longer interval between neoadjuvant CRT and surgery, and it represents the first attempt to evaluate response by measuring the radiological and pathological response by quantitative measures. It is of concern that, as is well known, there are patients who do not respond to neoadjuvant CRT, in our study $18.5 \%$. In our exploratory study nonresponders did not have worse outcome after successful surgery, however no sensible clinician would risk compromising those patients with a longer interval and, as a matter of fact, it would be necessary to operate on them as soon as it is established there is no response. This could be immediately after the end of CRT or even within its course if we are able to establish ways to identify them in time.

A similar dilemma exists with the decision as to what is the ideal moment to interrupt deferment of surgery. We recognize the limitations of this pilot study, including its retrospective nature as well as the limited number of cases, which do not allow us to draw more robust conclusions. The waiting time post CRT should be the subject of further research, along with the effort to use biomarkers as prognostic tools to distinguish between good and poor responders to CRT.

\section{Statement of Ethics}

The authors confirm that this study was conducted according to the local King's College Hospital ethics policy for retrospective studies and in accordance with the Helsinki Declaration of 1975. Informed written consent was obtained prior to every medical action in our academic institution, King's College Hospital, for publication of patient data for scientific reasons.

\section{Disclosure Statement}

The authors declare no conflicts of interest. They have no financial or nonfinancial competing interests (political, personal, religious, ideological, academic, intellectual, commercial, or any other) to declare in relation to this paper. No funding was obtained for the writing of this paper.

\section{Author Contributions}

A. Plastiras contributed to data collection and analysis as well as drafting of the manuscript. M. Sideris contributed to data analysis and drafting of parts of the manuscript. S. Papagrigoriadis is the senior author of the study who conceived the study, interpreted the data, edited the manuscript, and operated on many of these patients. A. Gaya is the oncologist supervisor of the study. A. Haji, J. Nunoo-Mensah, and A. Haq operated on many of these patients, contributed to the editing of the manuscript, and provided feedback on the interpretation of the data. All authors gave consent for submission of this article. 


\section{References}

1 De Campos-Lobato LF, Geisler DP, da Luz Moreira A, Stocchi L, Dietz D, Kalady MF: Neoadjuvant therapy for rectal cancer: the impact of longer interval between chemoradiation and surgery. J Gastrointest Surg 2011;15: 444-450.

2 Pahlman L: Optimal timing of surgery after preoperative chemoradiotherapy for rectal cancer. Nat Clin Pract Oncol 2009;6:128-129.

3 Colorectal Cancer Collaborative Group: Adjuvant radiotherapy for rectal cancer: a systematic overview of 8,507 patients from 22 randomized trials. Lancet 2001;358:1291-1304.

4 Van Gijn W, Marijnen CA, Nagtegaal ID, Kranenbarg EM, Putter H, Wiggers T, et al: Preoperative radiotherapy combined with total mesorectal excision for resectable rectal cancer: 12-year follow-up of the multicentre, randomised controlled TME trial. Lancet Oncol 2011;12:575-582.

5 Francois Y, Nemoz CJ, Baulieux J, Vignal J, Grandjean JP, Partensky C, et al: Influence of the interval between preoperative radiation therapy and surgery on downstaging and on the rate of sphincter-sparing surgery for rectal cancer: the Lyon R90-01 randomized trial. J Clin Oncol 1999;17:2396.

6 Habr-Gama A, Perez RO, Proscurshim I, Nunes Dos Santos RM, Kiss D, Gama-Rodrigues J, et al: Interval between surgery and neoadjuvant chemoradiation therapy for distal rectal cancer: does delayed surgery have an impact on outcome? Int J Radiat Oncol Biol Phys 2008;71:1181-1188.

7 Kodner IJ, Shemesh EI, Fry RD, Walz BJ, Myerson R, Fleshman JW, et al: Preoperative irradiation for rectal cancer. Improved local control and long-term survival. Ann Surg 1989;209:194-199.

8 Sauer R, Liersch T, Merkel S, Fietkau R, Hohenberger W, Hess C, et al: Preoperative versus postoperative chemoradiotherapy for locally advanced rectal cancer: results of the German CAO/ARO/AIO-94 randomized phase III trial after a median follow-up of 11 years. J Clin Oncol 2012;30:1926-1933.

9 Randomized study on preoperative radiotherapy in rectal carcinoma. Stockholm Colorectal Cancer Study Group. Ann Surg Oncol 1996;3:423-430.

10 Park IJ, You YN, Agarwal A, Skibber JM, Rodriguez-Bigas MA, Eng C, et al: Neoadjuvant treatment response as an early response indicator for patients with rectal cancer. J Clin Oncol 2012;30:1770-1776.

11 Johnston DF, Lawrence KM, Sizer BF, Arulampalam TH, Motson RW, Dove E, et al: Locally advanced rectal cancer: histopathological correlation and predictive accuracy of serial MRI after neoadjuvant chemotherapy. Br J Radiol 2009;82:332-336.

12 Kerr SF, Norton S, Glynne-Jones R: Delaying surgery after neoadjuvant chemoradiotherapy for rectal cancer may reduce postoperative morbidity without compromising prognosis. Br J Surg 2008;95:1534-1540.

13 Tran CL, Udani S, Holt A, Arnell T, Kumar R, Stamos MJ: Evaluation of safety of increased time interval between chemoradiation and resection for rectal cancer. Am J Surg 2006;192:873-877.

14 Garcia-Aguilar J, Smith DD, Avila K, Bergsland EK, Chu P, Krieg RM, et al: Optimal timing of surgery after chemoradiation for advanced rectal cancer: preliminary results of a multicenter, nonrandomized phase II prospective trial. Ann Surg 2011;254:97-102.

15 Tulchinsky H, Shmueli E, Figer A, Klausner JM, Rabau M: An interval >7 weeks between neoadjuvant therapy and surgery improves pathologic complete response and disease-free survival in patients with locally advanced rectal cancer. Ann Surg Oncol 2008;15:2661-2667.

16 Marijnen CA, Kapiteijn E, van de Velde CJ, Martijn H, Steup WH, Wiggers T, et al: Acute side effects and complications after short-term preoperative radiotherapy combined with total mesorectal excision in primary rectal cancer: report of a multicenter randomized trial. J Clin Oncol 2002;20:817-825.

17 Guillem JG, Chessin DB, Cohen AM, Shia J, Mazumdar M, Enker W, et al: Long-term oncologic outcome following preoperative combined modality therapy and total mesorectal excision of locally advanced rectal cancer. Ann Surg 2005;241:829-836.

18 Onaitis MW, Noone RB, Hartwig M, Hurwitz H, Morse M, Jowell P, et al: Neoadjuvant chemoradiation for rectal cancer: analysis of clinical outcomes from a 13-year institutional experience. Ann Surg 2001;233:778-785.

19 Shivnani AT, Small W Jr, Stryker SJ, Kiel KD, Lim S, Halverson AL, et al: Pre-operative chemoradiation for rectal cancer: results of multimodality management and analysis of prognostic factors. Am J Surg 2007;193:389393.

20 Kim NK, Baik SH, Seong JS, Kim H, Roh JK, Lee KY, et al: Oncologic outcomes after neoadjuvant chemoradiation followed by curative resection with tumor-specific mesorectal excision for fixed locally advanced rectal cancer: impact of post irradiated pathologic downstaging on local recurrence and survival. Ann Surg 2006; 244:1024-1030.

21 Fang CB, Gomes CM, Formiga FB, Fonseca VA, Carvalho MP, Klug WA: Is the delayed surgery after neoadjuvant chemoradiation beneficial for locally advanced rectal cancer? Arq Bras Cir Dig 2013;26:31-35.

22 Panagiotopoulou IG, Parashar D, Qasem E, Mezher-Sikafi R, Parmar J, Wells AD, et al: Neoadjuvant long-course chemoradiotherapy for rectal cancer: does time to surgery matter? Int Surg 2015;100:968-973.

23 Li J, Liu H, Yin J, Liu S, Hu J, Du F, et al: Wait-and-see or radical surgery for rectal cancer patients with a clinical complete response after neoadjuvant chemoradiotherapy: a cohort study. Oncotarget 2015;6:42354-42361. 